\title{
A Spark-based genetic algorithm for sensor placement in large scale drinking water distribution systems
}

\author{
Chengyu Hu${ }^{1} \cdot$ Guo Ren ${ }^{1} \cdot$ Chao Liu' ${ }^{1} \cdot \operatorname{Ming} \mathrm{Li}^{2} \cdot$ Wei Jie $^{3}$
}

\begin{abstract}
Water pollution incidents have occurred frequently in recent years, causing severe damages, economic loss and long-lasting society impact. A viable solution is to install water quality monitoring sensors in water supply networks (WSNs) for real-time pollution detection, thereby mitigating the risk of catastrophic contamination incidents. Given the significant cost of placing sensors at all locations in a network, a critical issue is where to deploy sensors within WSNs, while achieving rapid detection of contaminant events. Existing studies have mainly focused on sensor placement in water distribution systems (WDSs). However, the problem is still not adequately addressed, especially for large scale WSNs. In this paper, we investigate the sensor placement problem in large scale WDSs with the objective of minimizing the impact of contamination events. Specifically, we propose a two-phase Spark-based genetic algorithm (SGA). Experimental results show that SGA outperforms
\end{abstract}

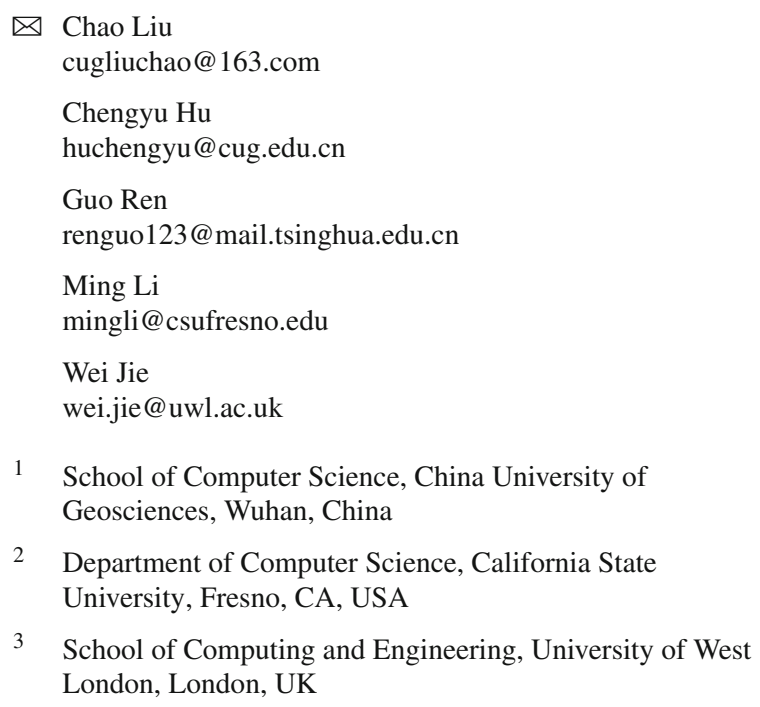

2 Department of Computer Science, California State University, Fresno, CA, USA

3 School of Computing and Engineering, University of West London, London, UK

other traditional algorithms in both accuracy and efficiency, which validates the feasibility and effectiveness of our proposed approach.

Keywords Sensor placement - Water distribution system . Genetic algorithm $\cdot$ Spark

\section{Introduction}

Clean drinking water is a critical resource for the health and well-being of all humans. However, water quality can easily deteriorate because of malicious attack or accidental incident. For example, an outbreak of waterborne disease epidemic in Walkerton, Ontario, Canada, in 2000 affected 2,300 people as a result of exposure to contaminated drinking water [1]. An Event such as the poisoning of water supply caused 71 people poisoned happened in Ruyang city, Henan Province, China in 2007 [2].

Deploying water quality sensor networks is considered as a viable approach for detecting contamination incidents in drinking WDS $[3,4]$. However, high cost of water quality sensors (e.g., a Hach chlorine sensor costs between USD 3000-5000) and installation/maintenance, as well as optimization methods, limit the number of sensor [5]. How to quickly detect the contaminant event in a large scale WDS with a fixed number of water quality sensors is a critical problem.

In the past decade, although SPP in drinking WDS has been well investigated, there are two main problems for deploying sensors in a large scale WSN (e.g., the number of node is greater than 10,000):

- Computational complexity. SPP is divided into two different constrained optimization problems: one is a 
sensor-constrained optimization problem, i.e. allowing only a fixed number of sensors; the other is a timeconstrained optimization problem, i. e. requiring contamination detection within a given time limit. Some researchers have proved that the two problems are NPhard or NP-Complete [6], and the former is polynomially equivalent to the asymmetric k-center problem, the latter is polynomially equivalent to the dominating set problem. Similarly, Carr et al. [7] formulated SPP as a mixed-integer program and proved that its complexity is NP-hard when the objective coefficients are not known with certainty.

- Large computation overhead. Drinking WDS in the real world is a complexity system which consists of thousands of miles of pipes, valves and pumps, which results in significant computational efforts for water quality simulation. As an example WSN shown in the battle of the water sensor network has 12,523 nodes, 2 sources, 2 tanks, 14822 pipes, 4 pumps, 5 valves [8]. Large scale WSN results in expensive computation overhead and storage requirement. For a large scale network with about 10,000 nodes, if each node has a probability of being injected contaminant, with the sampling time of the sensor being $10 \mathrm{~min}$ and the whole simulation time being $72 \mathrm{~h}$, then we need to simulate $10,000 \times 72 \times 60 \div 10=$ 4.32 million contaminant scenarios. If we consider that storing the contaminant concentration at each node needs 4 byte for each scenario, then the requirement of storage is 172.8 Gigabytes. Considering the running time of simulation on this network (roughly $4 \mathrm{~s}$ on a current Pentium $4.3 \mathrm{GHz}$ ), the time for an exhaustive simulation of all 4.32 million scenarios would require roughly 200 days [9].

To deal with computational complexity of SPP, some researchers applied some rule-based or graph-based methods to avoid the dilemma. For example, Chang et al. [10] employed two rules (e.g., accessibility and complexity rules) to generate a set of sensor placement locations. These methods do not need to simulate hydraulic and quality transport model, therefore incurring little computation and storage overhead. However, these methods seem to be too crude to locate sensors because they generally do not take into account the transport process of drinking water in WSN. In fact, for NP-hard or NP-Complete problems, evolutionary algorithms are a set of competent methods to get a near-optimal or optimal solution $[11,12]$. However, evolutionary algorithms often lead to longer computation. A big challenge is how to quickly and efficiently generate a near-optimal solution.

In recent years, there are a few research works on WDS involved in high performance computation. SPP is dealt with by the combination of parallel processing technique and evolutionary computation. Laszewski et al. [13] solved the contaminant source identification (CSI) problem on the super-computing resources of TeraGrid. Wu et al. [14] investigated the use of cloud computing in WDSs, where a pump scheduler has been deployed onto the high performance computer, through which a user can submit, execute and retrieve optimization analysis jobs. Shen et al. [15] applied parallel computing to simulate intrusion events for CSI problem with a super-computer. Wang et al. [16] presented a parallel method for the first time using the MapReduce paradigm to identify the contamination source in WDS.

To the best of our knowledge, there is no method that has been proposed in literatures for SPP using Spark to parallelize genetic algorithm (GA). Herein, as a first attempt, we use Spark to implement two-phase genetic algorithm. The first phase is fitness evaluation parallelization, and the second phase is genetic operations. More specifically, the contributions of our work are as follows:

- We present the system model and formulate SPP into a mixed-integer programming problem that satisfies the goal of minimizing the average time of detecting the contaminant events.

- We propose a two-phase genetic algorithm based on Spark model for sensor placement in drinking WDS.

- We give a comprehensive evaluation of the proposed algorithm. Through experiments, we verify the performance and effectiveness of our proposed algorithm.

The rest of the paper is organized as follows. Section 2 reviews the state of arts. Section 3 focuses on system model and mathematic formulation. Section 4 presents our proposed algorithm. Section 5 discusses simulation results. Section 6 concludes the paper and puts forward some issues which need to be further investigated in the future.

\section{Related work}

Sensor placement is to deploy water quality sensor in WSN, with the purposes of protecting municipal people against both deliberate and accidental hazard's intrusions. The physical structure of a water distribution system is a network in which nodes represent water sources, tanks, and junctions.

In a typical scenario shown in Fig. 1, two perfect sensors are deployed at junction 30 and junction 118, reservoir is located at node 129, and two tanks are at nodes 130 and 131. The contaminant is injected at the node 18 from 7 a.m. to 9 a.m. with an injection flow rate of $120 \mathrm{~L} / \mathrm{h}$, contaminant concentration of $230,000 \mathrm{mg} / \mathrm{L}$. After the injection, the contaminant gradually dilutes with the water flows from the upstream to downstream. After $2 \mathrm{~h}$, the contaminant will pass through the node 118 where one quality sensor stands, and then a warning alarm will be raised. 
Fig. 1 A WDS with 126 nodes, 1 source, 2 tanks, 168 pipes, 2 pumps and 8 valves

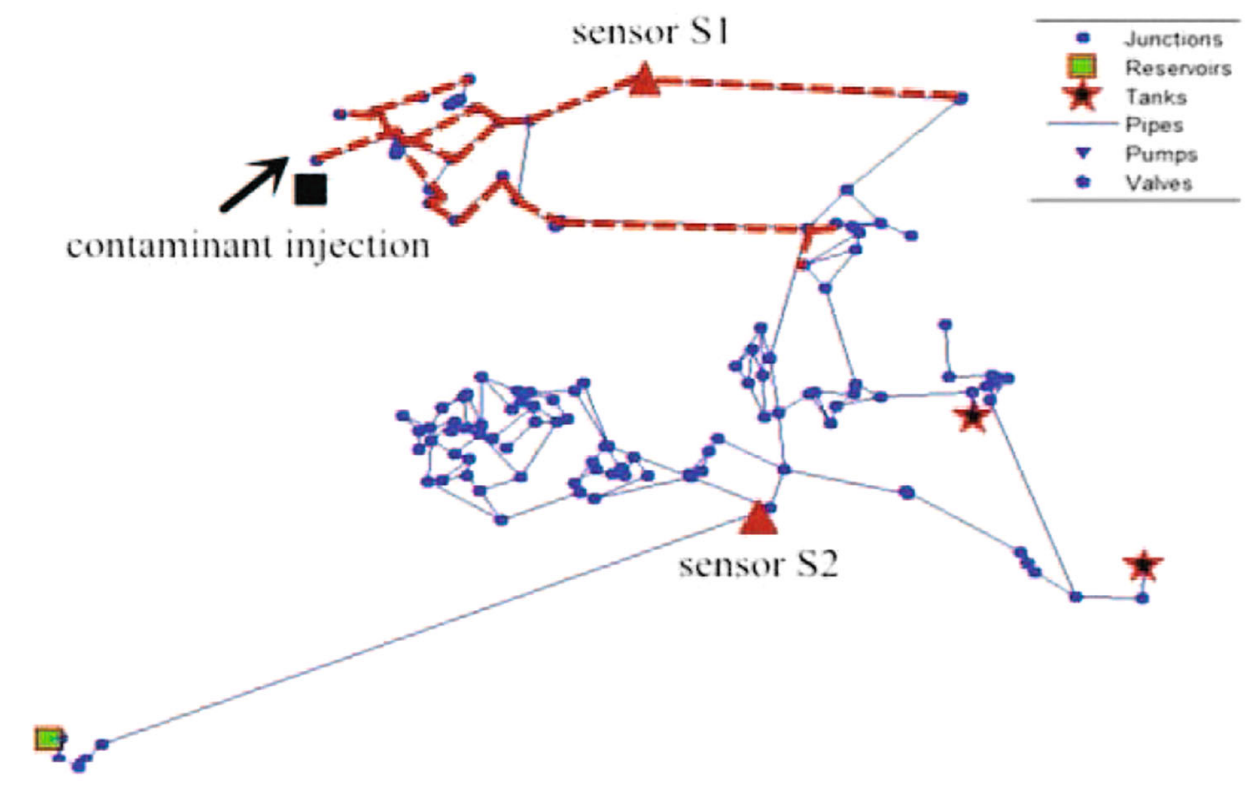

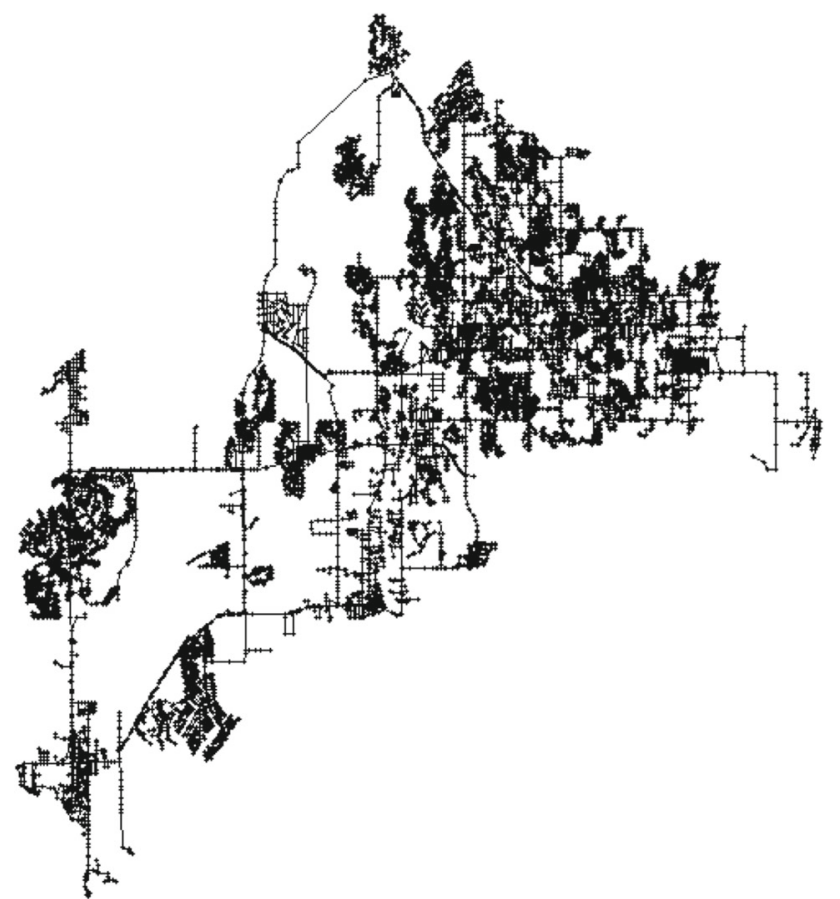

Fig. 2 Placement of Network2 in BWSN (12,523 nodes, 2 sources, 2 tanks, 14,822 pipes, 4 pumps, 5 valves) [8]

In Fig. 1, we are mainly concerned with which node should be deployed with sensors. For a small or medium scale WSN, it is easy to give the optimal placement of sensors by exhaustive search. However, it is a difficult task to obtain the optimal sensor placement for a large scale WSN. As shown in the example of Fig. 2, if we deploy 20 sensor to the WSN with 10,000 nodes, there are $C_{10,000}^{20}$ placement schemes of sensors.

\subsection{Some approaches for sensor placement problem in WDS}

For the SPP in WDS, a variety of different sensor placement optimization algorithms have been used to address the problem. They includes deterministic or heuristic algorithm, such as integer programming, genetic algorithm, simulated annealing and ant colony optimization [17].

As a high dimensional combination optimization problem, SPP is too computationally-intensive to find an exact solution. However, sometimes a near-optimal solution can be sufficient. Therefore, some effective heuristic random algorithms such as evolutionary techniques should be explored.

Evolutionary algorithms are typically used to provide good approximate solutions to problems that cannot be solved easily using other techniques, such as graph classification applications $[18,19]$. Due to their random nature, evolutionary algorithms are never guaranteed to find an optimal solution for any problem, but they will often find a good solution if the one exists.

Guan et al. [20] first proposed a genetic algorithm simulation optimization methodology based on a single objective function approach in which the four quantitative design objectives were embedded. Liu and Pierre [21] used a multiobjective genetic algorithm to optimize the placement of sensor networks. Schwartz et al. [22] presented a genetic algorithm to find the optimal sensor placement. Ant colony optimization algorithm was also used for the optimization of the position of water quality sensors [23].

However, the above mentioned approaches are limited to solving SPP in a small or medium scale WSN. As is well known, evolutionary algorithm is one kind of populationbased heuristic random algorithm which involves multiple 
iterative fitness evaluations. For large scale drinking WSNs, the solution space increases exponentially with the size of WSNs, which need much more fitness evaluations, thus causing high computation overhead.

To address the intensive computation challenge, an evolutionary algorithm in parallel is studied as an alternative way to improve both performance and quality of the solutions. Spark is an emerging popular parallel computation framework, and characterized by fast in-memory computing, high performance, good scalability. Genetic algorithm, as one of the evolutionary algorithms, requires a large number of iterative operations, and in line with the CPU-intensive computing features. Thus, it can benefit from Spark's in-memory computing ability. Our goal is to design a Spark-based parallel GA which is suitable for sensor placement [24].

\subsection{MapReduce and Spark}

As two very popular open source cluster and cloud computing frameworks for large scale data processing, MapReduce and Spark expose a simple programming API to users. But the major architectural components such as shuffle, execution model, and caching lead to different performance between MapReduce and Spark.

MapReduce is a popular programming model for Cloud computing and big data computing [25-27]. It exposes a simple programming API in terms of map and reduce functions. The simplicity of MapReduce is attractive for users, but the framework has several limitations. Applications such as machine learning and graph analytics iteratively process the data, which means multiple rounds of computation are performed on the same data. In MapReduce, every job reads its input data, processes it, and then writes it back to Hadoop Distributed File System (HDFS). For the next job to consume the output of a previously run job, it has to repeat the read, process, and write cycle. For iterative algorithms, which want to read once, and iterate over the data many times, the MapReduce model poses a significant overhead. As a typical iterative algorithm, genetic algorithm needs many times iterative computations for evaluation of fitness function. To overcome the above limitations of MapReduce, Spark uses resilient distributed datasets (RDDs) which implement inmemory data structures and cache intermediate data across a set of nodes [28]. Since RDDs can be kept in memory, algorithms can iterate over RDD data many times very efficiently. In the paper, we make a simple experiment by evaluating the fitness function as a computing task. In Figs. 3 and 4, the horizontal axis is the number of tasks, and the vertical axis is the execution time. We can see that each Mapreduce task takes about $120 \mathrm{~s}$, with $50 \mathrm{~s}$ for map and reduce operations and $70 \mathrm{~s}$ for shuffle and I/O jobs. In comparison, each Spark task takes only $3 \mathrm{~s}$ except for the first task, due to the first load data.

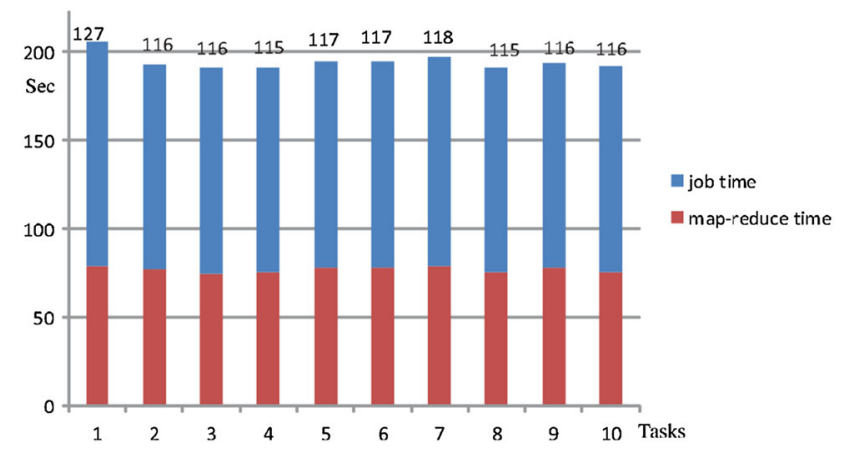

Fig. 3 Execution time of each task using MapReduce model

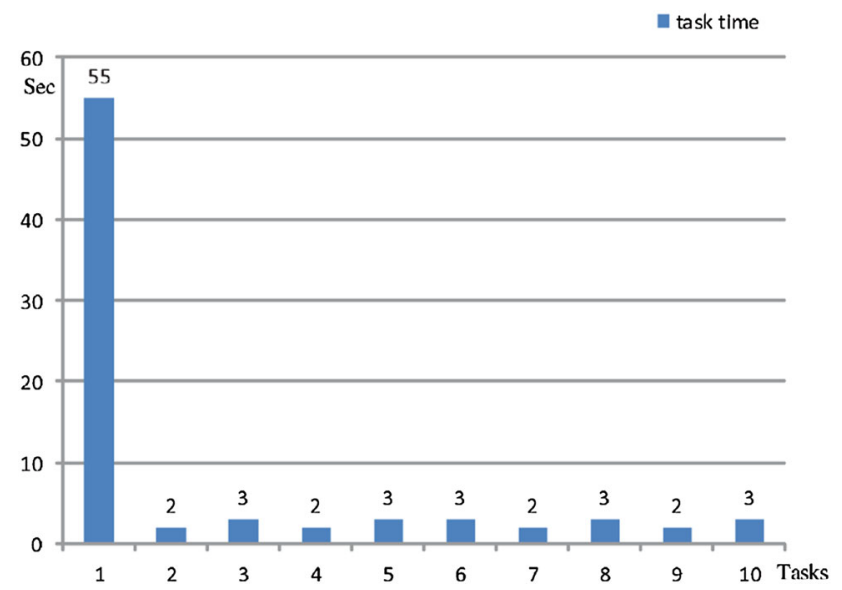

Fig. 4 Execution time of each task using Spark model

\section{System model and problem formulation}

\subsection{System model}

WDS, which consists of thousands of pipes, junctions and hydro-valves, may have a loop or branch network topology, or a combination of both. It is often modeled as a graph $G=(V, E)$, where vertices in $V$ represent junctions, tanks, or other sources, and edges in $E$ represent pipes, pumps, and valves. Drinking water flows are driven by pumping rates and pressure, which may vary frequently.

A contaminant event $a$ refers to the injection of poison substance by malicious attacks and may occur at any node, any time with uncertain dose of toxic chemicals or biological substance. The set of all possible contaminant scenarios $A$ is infinite because of uncertainty, a representative set of scenarios and quantify damages can be listed by enumeration or produced by random sampling.

In order to track how the contamination spread through the system, sensor placement problem requires the ability to perform hydraulic and quality simulation to evaluate the flow pattern at any time under the condition of known or 
assumed water demand. Most researchers rely on EPANET, a computer program developed by EPAs Water Supply and Water Resources Division as the main simulation tool [29].

For the convenience of analyzing sensor placement problem, We make the following assumptions.

- A contamination event occurs at a single point in the network.

- The contaminant is conservative, i. e., it does not react with the substance in the water.

- Sensors are fixed at the junctions and protect downstream populations. A population is considered exposed if it could be reached by a flow path from the contamination point without passing a sensor.

- Water quality sensors work with two-value measurement, which means only a discrete yes/no indication of contamination is available from these sensors.

- Sensors are perfect, which mean they can detect contaminants of any concentration with no false negatives and false positives; or they are capable to instantaneously detect a contaminant as soon as its concentration exceeds a minimum accepted value.

- A contamination warning is raised once a sensor detects a contamination event, and then response reactions are taken to isolate and flush contaminated water without any delay.

\subsection{Problem formulation}

Generally speaking, the objective of sensor placement in WDS is to detect contamination events and thus to mitigate the impact of contamination events. A typical mixed-integer programming (MIP) formulation for expected-impact sensor placement design is as follows.

$$
\min \sum_{a \in A} \alpha_{a} \sum_{i \in L_{a}} d_{a i} x_{a i}
$$

Constraint of equalities and inequalities are as follows:

$$
\begin{aligned}
& \sum_{i \in L_{a}} x_{a i}=1 \quad \forall a \in A \\
& x_{a i} \leq s_{i} \quad \forall a \in A, i \in L_{a} \\
& \sum_{i \in L} c_{i} s_{i} \leq p \\
& s_{i} \in\{0,1\} \quad \forall i \in L \\
& 0 \leq x_{a i} \leq 1 \quad \forall a \in A, i \in L_{a}
\end{aligned}
$$

This MIP minimizes the expected impact of a set of contamination incidents defined by $A$. For each incident $a \in A$, $\alpha_{a}$ is the weight of incident $a$, which is a probability of contaminant injection. $d_{a i}$ is the impact by contaminant event $a$ at node $i, L$ is a set of locations from the full set, where a location refers to a network node. For each incident $a, L_{a}$ is the set of locations that can be contaminated by an incident $a$.

$x_{a i}$ is the decision variable, which indicates whether incident $a$ is witnessed by a sensor at location $i$. It is defined as continuous variables between 0 and 1 . In practice, there is always an optimal solution where $x_{a i}$ is binary.

$s_{i}$ is a binary decision variable, $s_{i}=1$ indicates that a sensor are deployed at $i$ node. Collectively, all of the $s_{i}$ variables indicate where sensors are placed in the network; $c_{i}$ is the cost of placing a sensor at location $i$, and $p$ is the budget. If the sensors are isomorphic, then $p$ is a fixed number.

The constraints of Eq. (2) assures that one sensor will send alerts for each incident. The constraints of inequalities (3) forbids a location from sending alerts if there is no sensor installed there. The third set of constraints enforces the limited budget for the total number of sensors.

Ostfeld et al. [8] defined four quantitative design objectives as contamination impact such as average detection time, average population affected prior to detection, average consumption of contaminated water prior to detection, and average detection likelihood. These different objectives functions can be described by the impact $d_{a i}$. In the paper, we use average detection time as the performance criterion. That is, the lesser the average detection time is, the quicker we can detect the contaminant. Minimum average detection time can be defined as Eq. (7):

$\min \alpha_{a} \sum_{a \in A} T_{i \in L_{a}}\left(x_{a i}\right)$

where, $T_{i \in L_{a}}\left(x_{a i}\right)$ is the minimum detection time when the incident $a$ occurs and $L$ nodes are deployed with sensors. Ideally, if the number of sensors is enough to deploy at each node in WSN, then they will give the earliest alarm to the full population as soon as the contaminants are injected into the WSN.

\section{Algorithm design}

\subsection{Optimization framework}

Before using optimization algorithm to find the optimal placement of sensors, we need to make preprocessing or data preparation for sensor placement optimization. Herein, the 


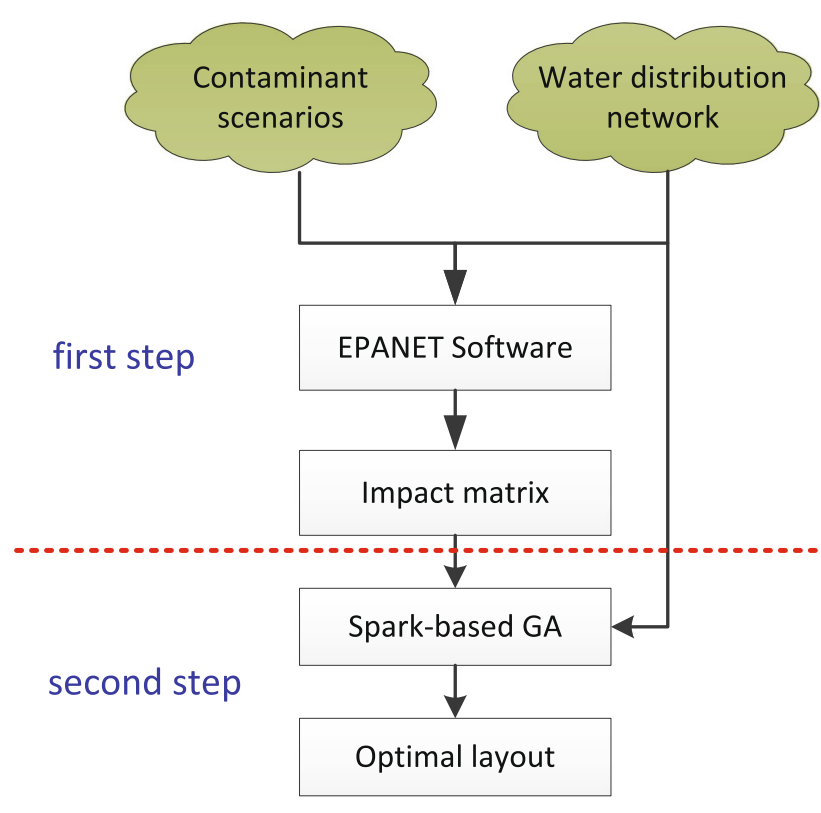

Fig. 5 Framework of optimization for SPP

first step is to simulate contamination incidents and compute contamination impact by the simulator EPANET [29], which is used to perform simulation of the hydraulic and water quality behavior within pressurized pipe network. Generally speaking, this process is time-consuming because contamination incidents are simulated for all network junctions, one for each hour of the day. After the simulation, the results of risk are stored in an impact matrix, and then the optimization algorithm can search the optimal placement of sensors according to the impact matrix. The framework of our method is shown in Fig. 5.

From Fig. 5, we can see that optimization process for SPP can be divided into two steps. The first step is to generate the contaminant events and use simulator (EPANET) to compute the impact matrix. And the second step is using optimization algorithm to select an optimal placement of sensors from the potential solution set.

\subsection{Spark-based genetic algorithm}

Genetic algorithm is inherently parallel since its fitness evaluation and evolution process can be carried out concurrently. There are four parallel models for distribution algorithm: global model, distributed model, cellular model, and hybrid model [30].

As a master-slave distributed computing, Spark is suitable for the parallelization of the global and distributed model of genetic algorithm. Therefore, aiming to improve the performance and effectiveness of genetic algorithm, we use Spark to parallelize it. The proposed parallel genetic algorithm is based on Spark resilient distributed datasets. The whole pop- ulation is stored as RDD and is cached in memory, which accelerates the subsequent processing.

We roughly divide our proposed algorithm into two phases. In the first phase, we initialize population, parallelize it into different partitions of RDD, and then evaluate fitness function of each individual on different workers. In the second phase, we perform the genetic operation on each individual after the parallel fitness evaluation value is returned to the driver. Then, we check whether the stop condition is satisfied. If it's not met, the algorithm continues the fitness evaluation in Phase 1.

Algorithm 1 details the pseudo-code of our proposed approach. The required inputs include the impact matrix, the maximum generation, the size of population, the crossover rate and the mutation rate. The output is an optimal or near-optimal solution, which is the best placement of sensors.

As seen in Algorithm 1, the population is first initialized with popsize individuals, each individual is made up of $s$ sensors that are deployed in WSN, then they are cached into different partitions of populationRDD (line 1-4). Subsequently, SGA performs the evaluation of the fitness function in parallel (line 6-8), the population with fitness value are counted by the driver. Next, SGA enters the second phase and executes evolution operations, such as crossover and mutation. And then a roulette selection operation is carried out according to the fitness value of each individual (line 9-14). Finally, if the results meet the stop condition, we output the best individual. Otherwise, SGA returns to the fitness evaluation phase (line 5).

As the evaluation of fitness function is the most timeconsumed part, we implement the parallel algorithm on the Spark cluster. Algorithm 2 describes the pseudo-code of the process of fitness function evaluation.

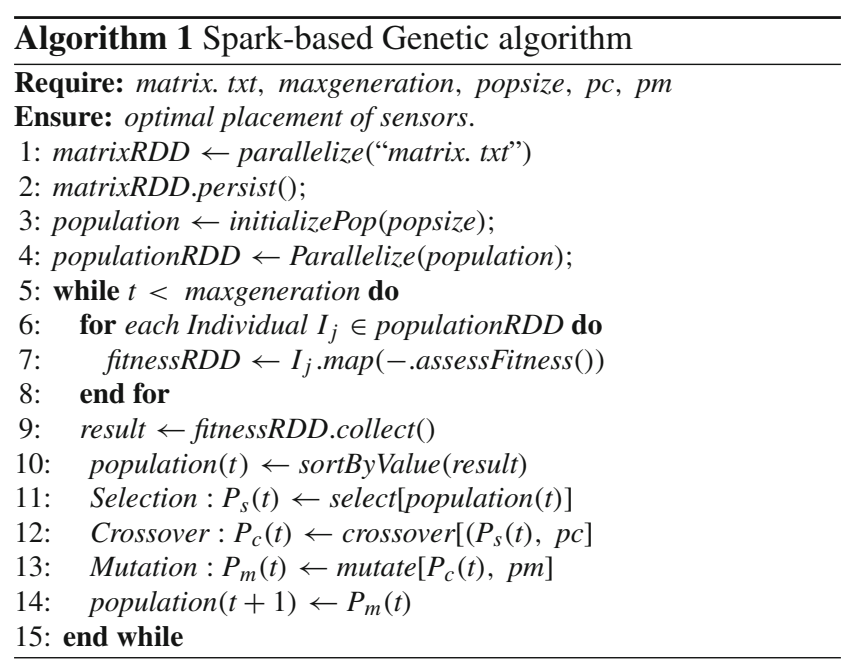




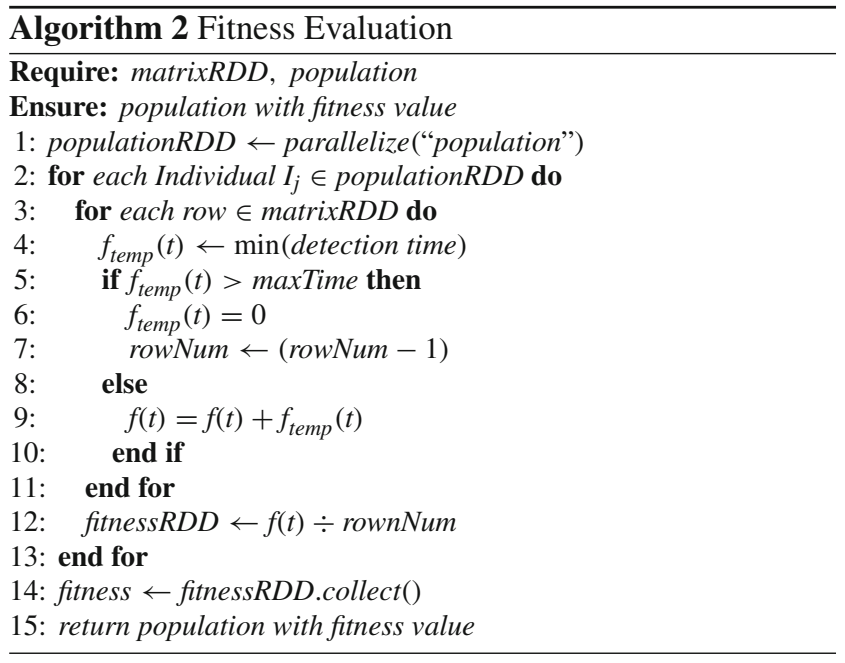

In Algorithm 2, the required inputs include the population and matrixRDD and the output is the population with fitness value. For the contaminant impact matrix, we first sort each rows and get the minimal detection time. Second, we sum up the minimal detection time for each rows and return the mean value as the fitness value (line 2-13). As the matrix has more than 25,000 rows and 10,000 columns, this part has the most significant computation overhead for our proposed SGA.

\section{Experiment results and analysis}

\subsection{Experiment settings}

In the experiment, we deploy 5 or 20 sensors in a large scale WSN as shown in Fig. 2. In order to evaluate the placement of sensors, we first simulate the contamination scenarios. Each contaminant intrusions occur at random time with an injection flow rate of $125 \mathrm{~L} / \mathrm{h}$, contamination concentration of $230,000 \mathrm{mg} / \mathrm{L}$, and injection duration of $2 \mathrm{~h}$. The contaminant was assumed conservative after injection. Each contamination scenario involved a single injection location, which may occur at any network node and begin at any time with equal probability. For purposes of design evaluation, contaminant concentrations were evaluated using a 5-min time step. A randomized impact matrix of 25,054 events (two injections at each node of the system, at two random times) was generated.

To verify the performance of SGA, we implemented SGA on a cluster with eight servers. Each server is equipped with a 2.0 GHZ dual-core processor and $16 \mathrm{~GB}$ memory, as summarized in Table 1.

The default parameters of SGA are listed in Table 2.

\subsection{Accuracy evaluation}

To test and evaluate the accuracy of SGA, we performed two categories of experiments with 5 and 20 sensors in water dis-
Table 1 Cluster configuration

\begin{tabular}{ll}
\hline Number of servers & 8 \\
Processor & $2.0 \mathrm{GHZ}$ \\
Memory & $16 \mathrm{~GB}$ \\
Operation System & Ubuntu 12.04 \\
Hadoop & Hadoop-2.4.0 \\
Spark & Spark-1.4.0 \\
\hline
\end{tabular}

Table 2 Spark-based Genetic Algorithm Settings

\begin{tabular}{ll}
\hline Crossover probability & 0.95 \\
\hline Mutation probability & 0.1 \\
Elite strategy & 10 \\
Population size & 80 \\
Evolution generation & 50 \\
\hline
\end{tabular}

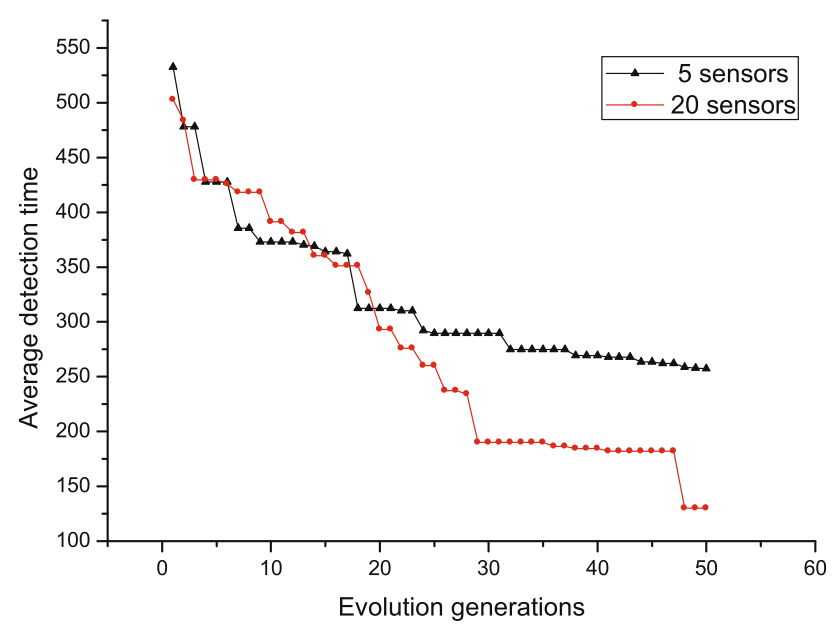

Fig. 6 Fitness value versus evolution generation, 5 sensors or 20 sensors are deployed in large scale WSN)

tribution network. Then we compared the performance with two other algorithms, i. e., the traditional genetic algorithm and the greedy algorithm [8].

Figure 6 shows that fitness function value decreases as the evolution generation increases from 1 to 50. The reason is that all of the three algorithms are convergent. In addition, it can be seen that the more sensors will lead to the reduction of average detection time. It should note that if we equip each node with one sensor, the average detection time is zero.

Tables 3 and 4 show the optimal sensor placement and the average detection time generated by the three algorithms, with 5 or 20 sensors in WSN respectively. It can be seen that our method can give a better placement of sensors which make the average detection time much smaller than the genetic algorithm and the greedy algorithm. 
Table 3 Solution for 5 sensors in a large WSN

\begin{tabular}{lll}
\hline Algorithm & Sensor location & Average detection time \\
\hline Genetic algorithm & $321,3770,4084,4939,7762$ & 795 \\
Greedy algorithm & $10874,4684,11304,3357,11184$ & 789 \\
SGA & $5240,5665,5883,6107,8897$ & 257 \\
\hline
\end{tabular}

Table 4 Solution for 20 sensors in a large WSN

\begin{tabular}{llr}
\hline Algorithm & Sensor location & Average detection time \\
\hline Genetic algorithm & $174,311,1486,1905,2589,2991,3548,3757,3864,4184,4238$ & \\
& $5091,6995,7145,7689,8826,9308,9787,10614,12086$ & 645 \\
Greedy algorithm & $10874,4684,11304,3357,11184,1478,9142,1904,4032,9364,4240$ & 665 \\
SGA & $4132,3635,2579,3836,6700,8999,3747,8834,3229$ & 130 \\
& $426,966,2934,5027,5206,5558,5858,6034,6117,6710,7010,7256$ & \\
\hline
\end{tabular}

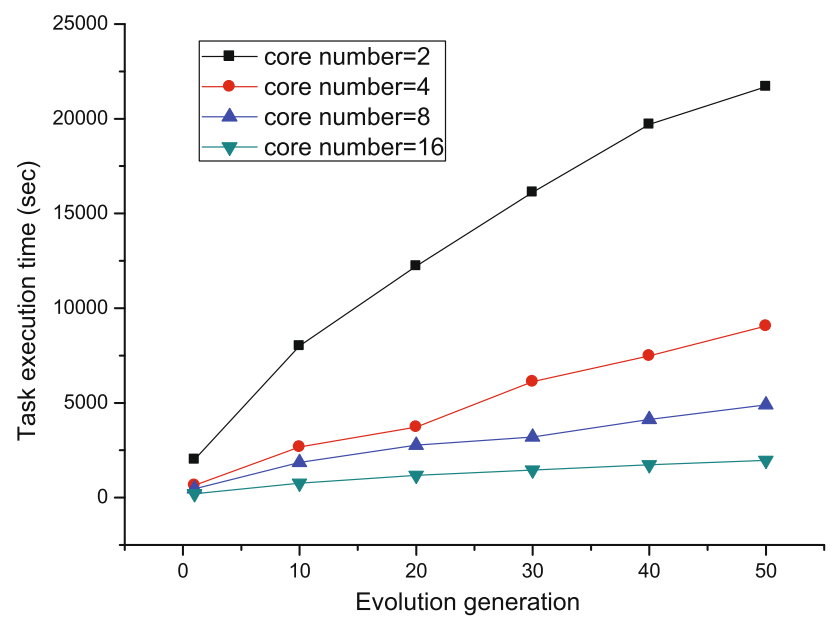

Fig. 7 Task execution time versus the number of evolution generation, 20 sensors are deployed in water distribution networks)

\subsection{Efficiency evaluation}

After showing the accuracy of our SGA, we further evaluate its efficiency in the metric of task execution time as well as the derived fitness value.

We first varied the generation number under cluster size (i. e., the number of CPU cores) 2, 4, 8 and 16 to check how it affects the task execution time in the metric of seconds. Figure 7 shows the task execution time under different generation numbers ranging from 1 to 50. It can be first seen that the task execution time is proportional to the generation number in SGA. This is simply because larger generation numbers require longer evolution time, which further incurs longer task execution time. On the other hand, we can also see that larger cluster size also indicates shorter task execution time. This implies that SGA efficiently explores the available cloud resources.

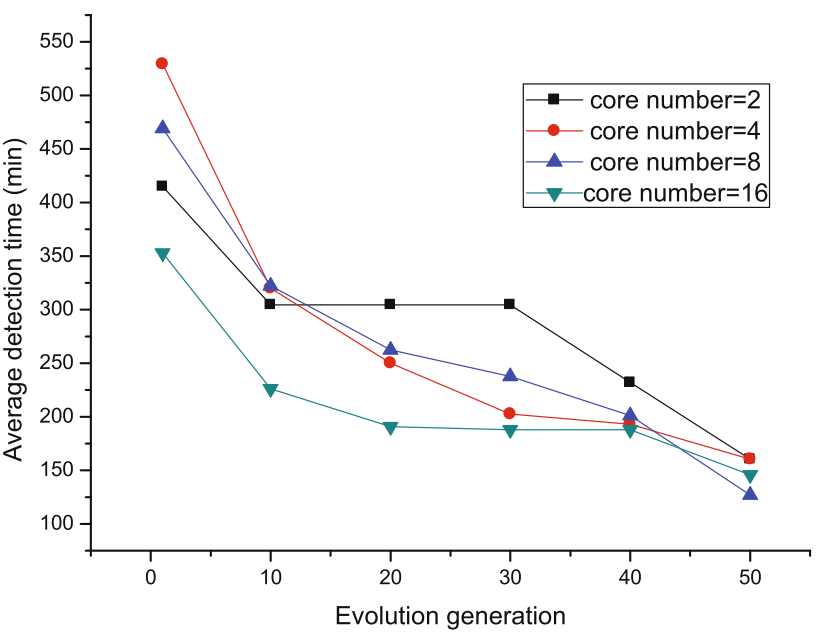

Fig. 8 Average detection time versus evolution generation, 20 sensors are deployed in large scale water distribution networks)

Next, we investigated how the generation number affects the optimization objective, i.e., average detection time. The results are shown in Fig. 8. On the contrary, we can see that increasing the generation number is beneficial to the fitness value. However, the benefit becomes marginal when the generation number is large enough. The nature of the evolution computation determines that the quality of the generation may finally converge. Although average detection time of four cases reduces with the increment of the number of generations, their rates of convergence are different because of the randomness of genetic algorithm.

Finally, we check how the cluster size influences the task execution time in the metric of speedup ratio Speedup, we define Speedup as the formula (8).

Speedup $=T_{1} / T_{p}$ 


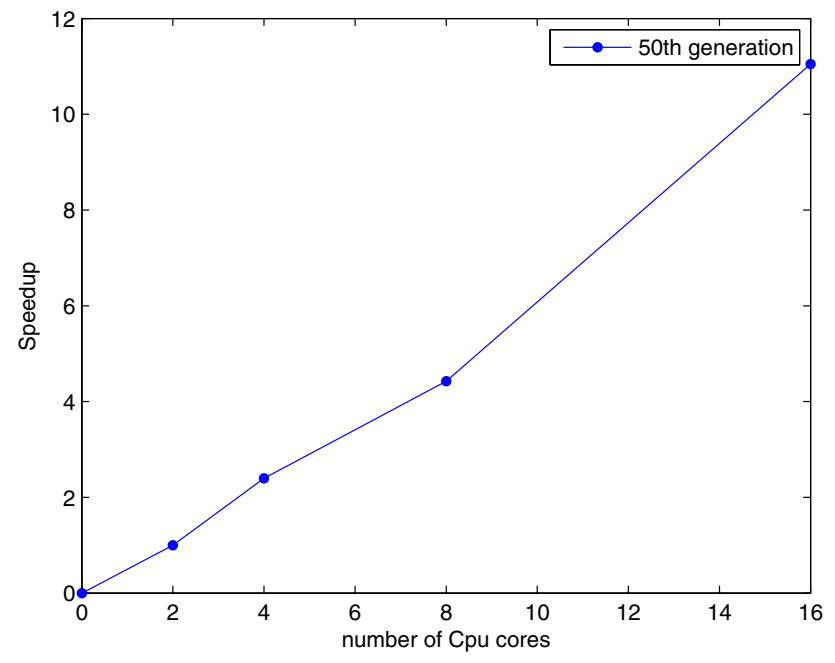

Fig. 9 Speedup versus the number of CPU cores

In formula (8), $T_{1}$ is the task execution time of one server with two CPU cores, and $T_{p}$ is the task execution time of cluster with $p$ CPU cores. Figure 9 presents evaluation results with the number of CPU cores ranging from 2 to 16 . We note that the speedup scales well with the cluster size.

\section{Conclusion}

In this paper, we proposed a Spark-based genetic algorithm for sensor placement in water distribution system. Based on the global and distributed model of GA Parallelization, SGA is divided into two phases. The first phase is the fitness evaluation that calculates each individual's fitness value on workers. The second phase is the genetic operations which run on the driver node. We evaluated the performance of SGA in terms of accuracy, efficiency and speedup. Results show that SGA outperforms the other two algorithms in the term of accuracy. We also find that SGA has a nearly linear speed-up for the parallel processing of sensor placement problem.

We have also identified a number of issues to be investigated in future studies with the following being noteworthy:

1. Investigation of the robustness of the proposed algorithm by applying it to a larger scale drinking water distribution networks and test it on a large size cluster.

2. Incorporation of the ability of sensors and accuracy of the hydraulic model into the proposed algorithm for realistic applications.

Acknowledgements This research was supported in part by the NSF of China (Grant No. 61305087, 61673354, 61672474, 61501412). Ming Li's research is partially supported by US National Science Foundation Award (Grant No. 1626586). This paper has been subjected to Hubei Key Laboratory of Intelligent Geo-Information Processing within School of Computer Science, China University of Geosciences, Wuhan,
China, 430074. It was also supported by Open Research Project of The Hubei Key Laboratory of Intelligent Geo-Information Processing (KLIGIP201603, KLIGIP201607).

\section{References}

1. Hrudey, S., Payment, P., Huck, P., Gillham, R., Hrudey, E.: A fatal waterborne disease epidemic in Walkerton, Ontario: comparison with other waterborne outbreaks in the developed world. Water Sci. Technol. 47(3), 7-14 (2003)

2. Kou, J.: Drinking water reservoirs were poisoned in Ruyang City of Henan Province. http://news.china.com/zh_cn/social/ 1007/20031004/11549967.html

3. Hart, W.E., Berry, J.W., Boman, E.G., Phillips, C.A., Riesen, L.A., Watson, J.: Limited-memory techniques for sensor placement in water distribution networks. In: Learning and Intelligent Optimization. Springer, Berlin (2008)

4. Zeng, D., Gu, L., Lian, L., Guo, S., Yao, H., Hu, J.: On cost-efficient sensor placement for contaminant detection in water distribution systems. IEEE Trans. Ind. Inf. 11, 112-121 (2016)

5. Xu, J., Johnson, M.P., Fischbeck, P.S., Small, M.J., Vanbriesen, J.M.: Robust placement of sensors in dynamic water distribution systems. Eur. J. Oper. Res. 202(3), 707-716 (2010)

6. Bergerwolf, T.Y., Hart, W., Saia, J.: Discrete sensor placement problems in distribution networks. Math. Comput. Model. 42(13), 1385-1396 (2005)

7. Carr, R.D., Greenberg, H.J., Hart, W.E., Konjevod, G., Lauer, E., Lin, H., Morrison, T., Phillips, C.A.: Robust optimization of contaminant sensor placement for community water systems. Math. Program. 107(1-2), 337-356 (2006)

8. Ostfeld, A., Uber, J.G., Salomons, E., Berry, J.W., Hart, W.E., Phillips, C.A., Watson, J.-P., Dorini, G., Jonkergouw, P., Kapelan, Z., et al.: The battle of the water sensor networks (BWSN): a design challenge for engineers and algorithms. J. Water Resour. Plan. Manag. 134(6), 556-568 (2008)

9. Krause, A., Leskovec, J., Guestrin, C., VanBriesen, J., Faloutsos, C.: Efficient sensor placement optimization for securing large water distribution networks. J. Water Resour. Plan. Manag. 134(6), 516526 (2008)

10. Chang, N.-B., Prapinpongsanone, N., Ernest, A.: Optimal sensor deployment in a large-scale complex drinking water network: comparisons between a rule-based decision support system and optimization models. Comput. Chem. Eng. 43, 191-199 (2012)

11. Gong, W., Cai, Z.: Differential evolution with ranking-based mutation operators. IEEE Trans. Syst. Man Cybernet. 43(6), 2066-2081 (2013)

12. Yang, M., Li, C., Cai, Z., Guan, J.: Differential evolution with autoenhanced population diversity. IEEE Trans. Syst. Man Cybernet. 45(2), 302-315 (2015)

13. Von Laszewski, G., Wang, L., Wang, F., Fox, G.C., Mahinthakumar, G.K.: Threat detection in an urban water distribution systems with simulations conducted in grids and clouds. In: Proceedings of the Second International Conference on Parallel, Distributed, Grid and Cloud Computing for Engineering, Ajaccio, Corsica, France (2011)

14. Wu, Z., Khaliefa, M.: Cloud computing for high performance optimization of water distribution systems. In: Proceedings of Albuquerque, New Mexico, World Environmental and Water Resources Congress (2012)

15. Shen, H., McBean, E.A.: Application of parallel computing in data mining for contaminant source identification in water distribution systems. Can. Water Resour. J. 38(1), 34-39 (2013)

16. Wang, L., Chen, D., Liu, W., Ma, Y., Wu, Y., Deng, Z.: DDDASbased parallel simulation of threat management for urban water distribution systems. Comput. Sci. Eng. 16(1), 8-17 (2014) 
17. Rathi, S., Gupta, R.: A critical review of sensor location methods for contamination detection in water distribution networks. Water Qual. Res. J. Can. 50(2), 95-108 (2015)

18. Wu, J., Zhu, X., Zhang, C., Yu, P.S.: Bag constrained structure pattern mining for multi-graph classification. IEEE Trans. Knowl. Data Eng. 26(10), 2382-2396 (2014)

19. Wu, J., Pan, S., Zhu, X., Zhang, P., Zhang, C.: SODE: self-adaptive one-dependence estimators for classification. Pattern Recogn. 51, 358-377 (2016)

20. Guan, J., Aral, M.M., Maslia, M.L., Grayman, W.M.: Optimization model and algorithms for design of water sensor placement in water distribution systems. In: 8th Annual Symposium on Water Distribution Systems Analysis. Environmental and Water Resources Institute of ASCE (EWRI of ASCE) New York, pp. 1-16 (2006)

21. Liu, S., Auckenthaler, P.: Optimal sensor placement for event detection and source identification in water distribution networks. J. Water Supply 63(1), 51-57 (2014)

22. Schwartz, R., Lahav, O., Ostfeld, A.: Optimal sensor placement in water distribution systems for injection of chlorpyrifos. In: World Environmental and Water Resources Congress 2014@ sWater Without Borders. ASCE, pp 485-494 (2014)

23. Afshar, A., Mariño, M.A.: Multi-objective coverage-based aco model for quality monitoring in large water networks. Water Resour. Manag. 26(8), 2159-2176 (2012)

24. Ma, Y., Wang, L., Liu, D., Liu, P., Wang, J., Tao, J.: Generic parallel programming for massive remote sensing data processing. In: International Conference on Cluster Computing, pp 420-428 (2012)

25. Dean, J., Ghemawat, S.: MapReduce: simplified data processing on large clusters. In: Operating Systems Design and Implementation, vol. 51(1), pp 107-113. Prentice-Hall, Upper Saddle River (2008)

26. Wang, L., Tao, J., Ranjan, R., Marten, H., Streit, A., Chen, J., Chen, D.: G-Hadoop: Mapreduce across distributed data centers for dataintensive computing. Future Gen. Comput. Syst. 29(3), 739-750 (2013)

27. Zhao, J., Wang, L., Tao, J., Chen, J., Sun, W., Ranjan, R., Kolodziej, J., Streit, A., Georgakopoulos, D.: A security framework in GHadoop for big data computing across distributed cloud data centres. J. Comput. Syst. Sci. 80(5), 994-1007 (2014)

28. Zaharia, M., Chowdhury, M., Das, T., Dave, A., Ma, J., Mccauley, M., Franklin, M.J., Shenker, S., Stoica, I.: Resilient distributed datasets: a fault-tolerant abstraction for in-memory cluster computing. Technical Report (2012)

29. U.S. EPA: Tutorial threat ensemble vulnerability analysis—sensor placement optimization tool TEVA-SPOT graphical user interface, version 2.2.0 Beta, EPA-600-R-08-147. Office of Research and Development, National Homeland Security Research Center, vol. 1(1), pp. 1-183 (2009)

30. Luque, G., Alba, E.: Parallel Genetic Algorithms: Theory and Real World Applications, vol. 367. Springer, Berlin (2011) ests include: evolutionary algorithm, swarm intelligence and cloud computing. 